Eduardo Prieto

Figura 1. John Evelyn, sistema de calefacción centralizada por aire para un invernadero (1694).

Figura 2. John Soane, sistema de suelo radiante del Banco de Inglaterra en Londres (1799). Fuente: (Archivo John Soane, Londres).
Doctor Arquitecto por la Universidad Politécnica de Madrid y licenciado en Filosofía.

\section{Espacios homotérmicos Una historia de la calefacción central en la arquitectura del siglo XIX}

\author{
Keywords: architecture, central heating, environmental design, heterothermia, homothermia
}

Brought on by the Industrial Revolution, the breakthrough of centralized heating in its different versions (air, steam, or water) gave rise to a genuine revolution in architecture: the passage from heterothermia (space organized in a gradient around a single thermal focus) to homothermia (the isotropic space served by split thermal foci). This article discusses that revolution through three complementary analyses: of the origins of centralized heating systems; of the technical, social, and symbolic crises produced by the implantation of those systems in 19th-century spaces; and of the strategies that architects of the period used to accommodate the new machinery.

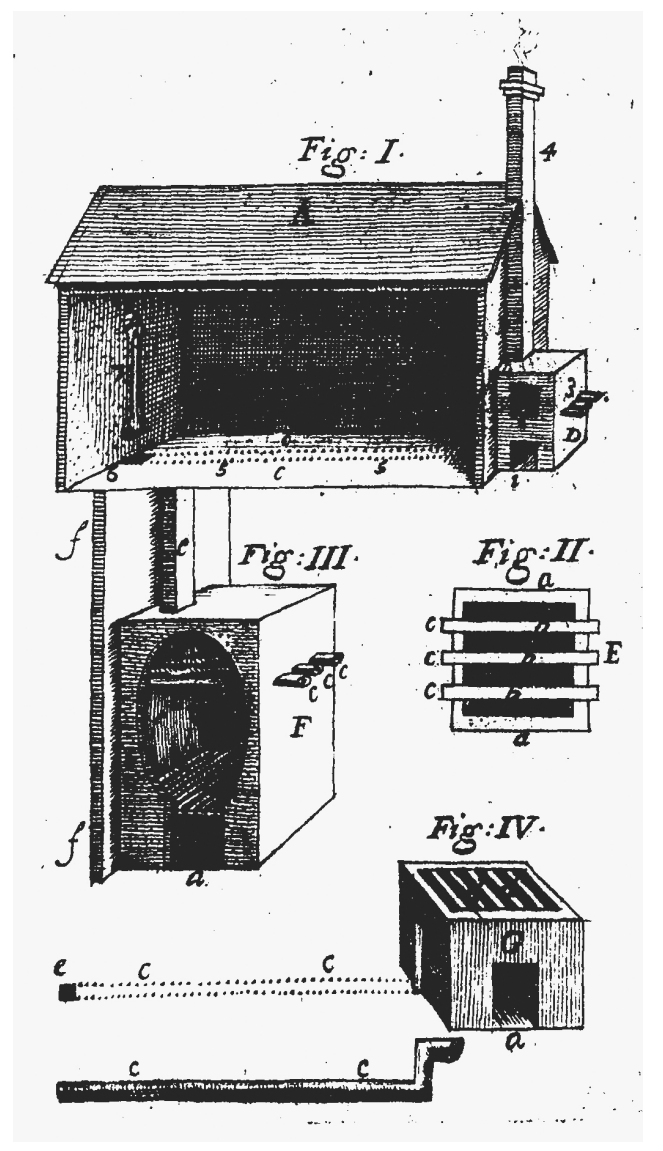

El origen de la calefacción moderna coin- cide con el de la Revolución Industrial, y se debe a una serie de invenciones que en su momento resultaron más bien inopinadas. A finales del siglo XVIII, los hogares con chimenea, perfeccionados gracias a las aportaciones de Benjamin Franklin y el conde Rumford, se habian introducido con tal fuerza en las casas aristocráticas y burguesas que se consideraban ya como el rasgo fundamental de una idea de comfort doméstico aún poco desarrollada y que se asoció a Gran Bretaña antes de implantarse en el resto de Europa. Es probable que los sistemas homotérmicos de calefacción centralizada nunca se hubieran desarrollado y perfeccionado, de no haber llegado la máquina de vapor y, con ella, la optimización del dominio termodinámico y la construcción de fábricas cada vez más grandes y con mayores exigencias higrotérmicas. La idea de un reparto de calor homogéneo y centralizado -la homotermia-, frente a la tradicional centrada en un foco y ligada al gradiente de temperatura -la heterotermia- tuvo que ver, en origen, con la necesidad de caldear procesos y espacios: los nuevos procesos productivos y los nuevos espacios laborales.

Es muy difícil determinar cuál fue el primero de los sistemas de calefacción centralizada moderna. Lo que sí puede decirse es que éstos tuvieron que ver con el perfeccionamiento de los viejos artefactos de calefacción por aire concebidos a la manera del hypocaustum romano. En 1694, el jardinero y escritor John Evelyn propuso caldear los invernaderos con un suelo radiante conectado a una estufa metálica (figura 1); solución que no se generalizó hasta finales del siglo XVIII en la Gran Bretaña que comenzaba a industrializarse, momento en el que la aplicaron en algunos de sus edificios arquitectos como Robert Adam y John Soane (figura 2). 
Con todo, el campo inicial de desarrollo de los sistemas centralizados por aire no fueron las viviendas ni los edificios públicos, sino las instalaciones industriales, como los molinos, las fábricas de algodón y las refinerias de azúcar o papel, donde se requería el aporte homogéneo de calor para favorecer el secado o la transformación de los materiales (Tann 1970: 109). En 1792, el ingeniero, empresario y reformador social William Strutt instaló una caldera metálica y una red centralizada de conductos de aire en el molino de una fábrica de algodón en Derby, cuya disposición resultaba muy semejante a la que, tal vez sin conocer el proyecto de Strutt, el ingeniero Oliver Evans describió poco después en un tratado sobre la construcción de molinos de agua publicado en Filadelfia en 1795 (Evans 1795: 369), (figura 3).

La calefacción de Strutt tuvo cierto éxito, y propició que los sistemas por aire se introdujeran tanto en molinos y fábricas cuanto en otro tipo de edificios menos previsibles. En 1810 se colocó, por primera vez, en un museo (el Hunterian de Glasgow), y ese mismo año en una casa, la del ingeniero y empresario Matthew Boulton, socio de James Watt (Tann 1970: 111). Sin embargo, donde la homotermia o distribución homogénea del calor a través del aire acabó encontrando su hábitat más propicio fue en los hospitales, enclaves en los que la obsesión higienista por las miasmas (en este caso, la obsesión por la insalubridad del humo) hizo de este tipo de sistemas una buena solución a la hora de combinar la calefacción y la ventilación natural. Así, el sistema de Strutt fue descrito con precisión anatómica por el ingeniero Charles Sylvester en un tratado de título revelador - The Philosophy of Domestic Economy-, publicado en 1809 y cuyo objeto fue presentar las innovaciones introducidas previamente en la calefacción del Hospital de Derbyshire (figura 4). ${ }^{1}$ Ésta consistía en una red de conductos enterrados por donde se hacía pasar el aire procedente del exterior y que, una vez 'precalentado' gracias al intercambio térmico con el suelo, se conectaba a un horno para acabar distribuyéndose, por diferencia de densidad y presiones, a través de una trama de conductos de fábrica de ladrillo distribuidos por el edificio de un modo sorprendentemente semejante a la manera en que se distribuyen hoy los conductos de instalaciones.

Con todo, fue un aristócrata e inventor francés afincado en Londres, el marqués de Chabannes, el que sacó todo el partido a la calefacción y a la ventilación natural que pro-

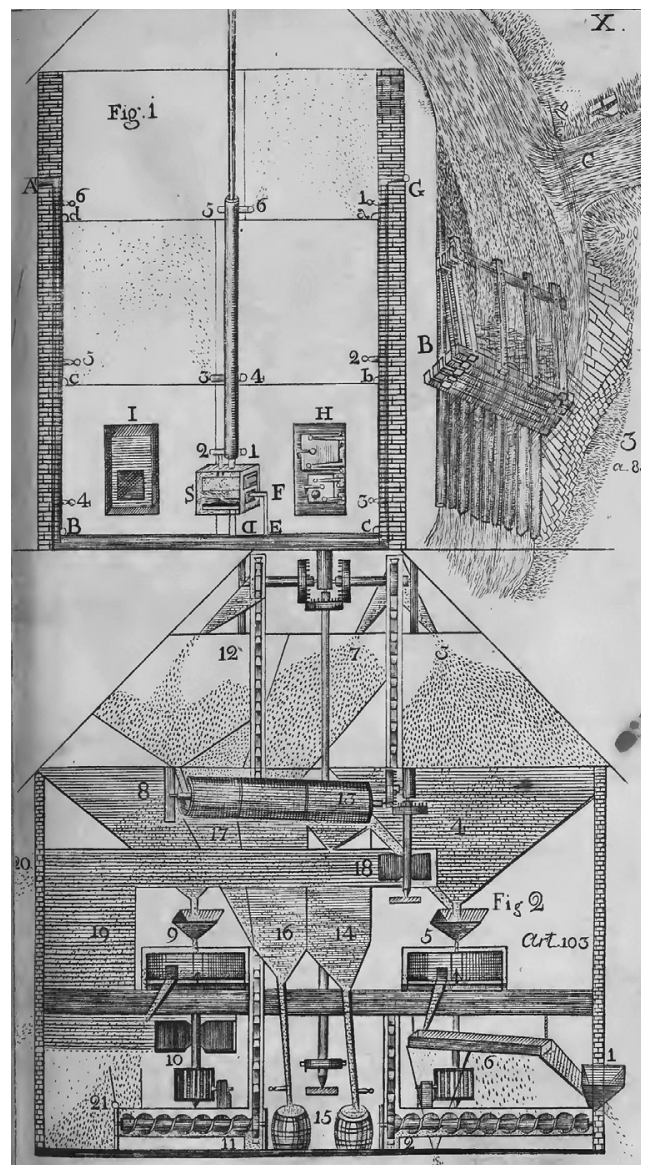

Figura 3. Oliver

Evans, sistema de calefacción centralizada por aire para un molino (1795). Fuente: (Evans 1795).

Figura 4. Charles Sylvester, sistema de calefacción centralizada por aire para la Derbyshire Infirmary (1809). Fuente: (Sylvester 1809).

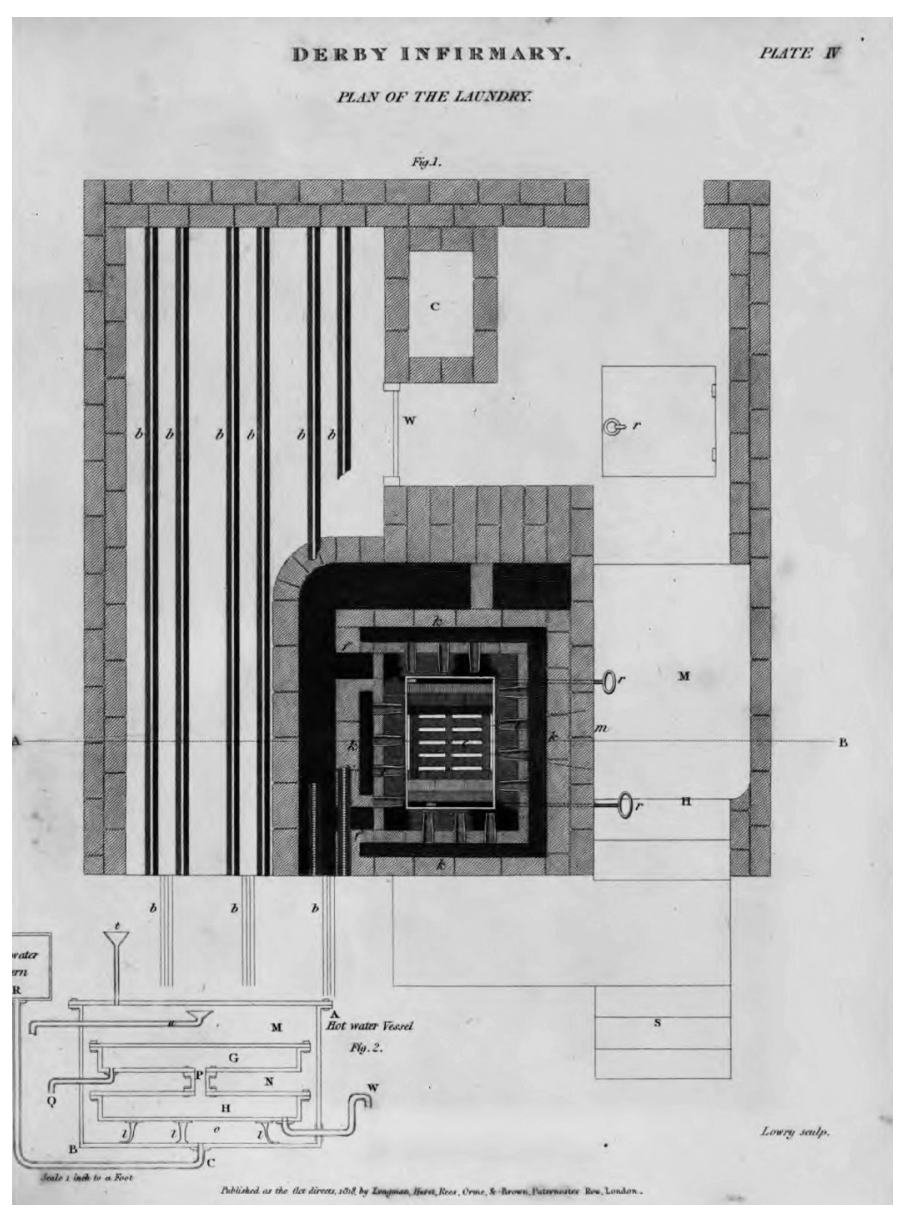


Figura 5. Neil Snodgrass, sistema de calefacción central por vapor (1806). Fuente: (Transactions, 1806). curaban los sistemas centralizados por aire. Chabannes se dio cuenta de que, desde el momento en que la ventilación afectaba a la temperatura de la habitación, no podía considerarse independiente de la calefacción, y en paralelo combatió el prejuicio, asociado a la teoria miasmática, de que el aire caliente era menos saludable que el frio, para proponer un conjunto de ingeniosas soluciones con el fin de favorecer lo que denominó, de manera pionera, la 'ventilación forzada': una ventilación cuyo sentido, en último término, no era sino la reforma social a través de la mejora de las condiciones ambientales. A pesar de todo, las innovaciones del marqués de Chabannes, más que el comienzo de un nuevo camino para los sistemas por aire, fueron el canto de cisne de todo un modo de generar confort. Térmicamente ineficiente y basada, en el fondo, en una contradicción (el hecho de caldear con un flujo caliente y ventilar con un flujo frío y siempre renovado), la calefacción por aire demostró tener poco futuro, aunque no dejara aún de aplicarse durante todo el siglo XIX, sobre todo en pequeñas instalaciones domésticas.

Más afortunadas fueron las instalaciones alimentadas por vapor, que no en vano se basaban en el uso de la fuerza motriz carac- terística de la época. De hecho, los orígenes de este tipo de calefacción están asociados a los pioneros de la Revolución Industrial: aunque en 1745 William Cook propuso conectar a una caldera de vapor un circuito cerrado y radiante, y en 1791 y 1793 John Hoyle y Joseph Green presentaron, respectivamente, sendas patentes del sistema (Tann 1970: 109), no fue hasta 1794 cuando James Watt y su socio, el ya mentado Matthew Boulton, proyectaron las primera instalación eficaz en la biblioteca del marqués de Landsdowne, a la que siguieron las colocadas, a partir de 1799, en varios molinos y fábricas de algodón (Bruegmann 1978: 146). ${ }^{2}$ Sin embargo, la primera versión científica y en verdad eficaz de las aplicaciones domésticas del sistema tuvo que esperar a 1806, cuando Neil Snodgrass recibió un premio de cuarenta guineas "for a mode of heating rooms by steam", ${ }^{3}$ que el inventor dibujó con detalle en la que quizá sea la primera representación arquitectónica de un sistema de calefacción centralizada convencional (figura 5).

Por ser tan eficiente en lo que toca a la distribución térmica, el sistema de vapor se desarrolló con rapidez. En 1810, el ingeniero Robertson Buchanan sistematizó los trazados, perfeccionó las uniones entre conductos

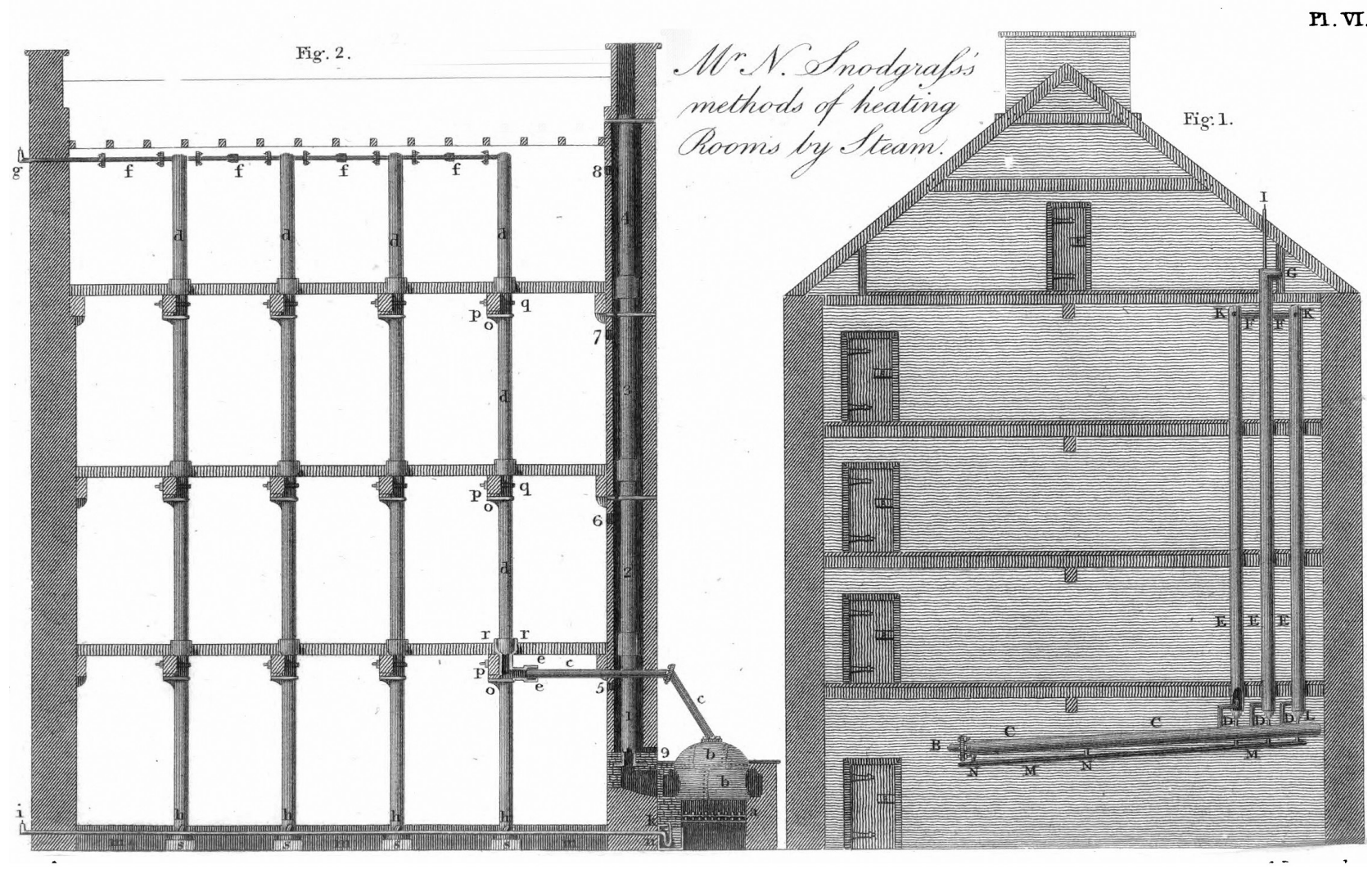




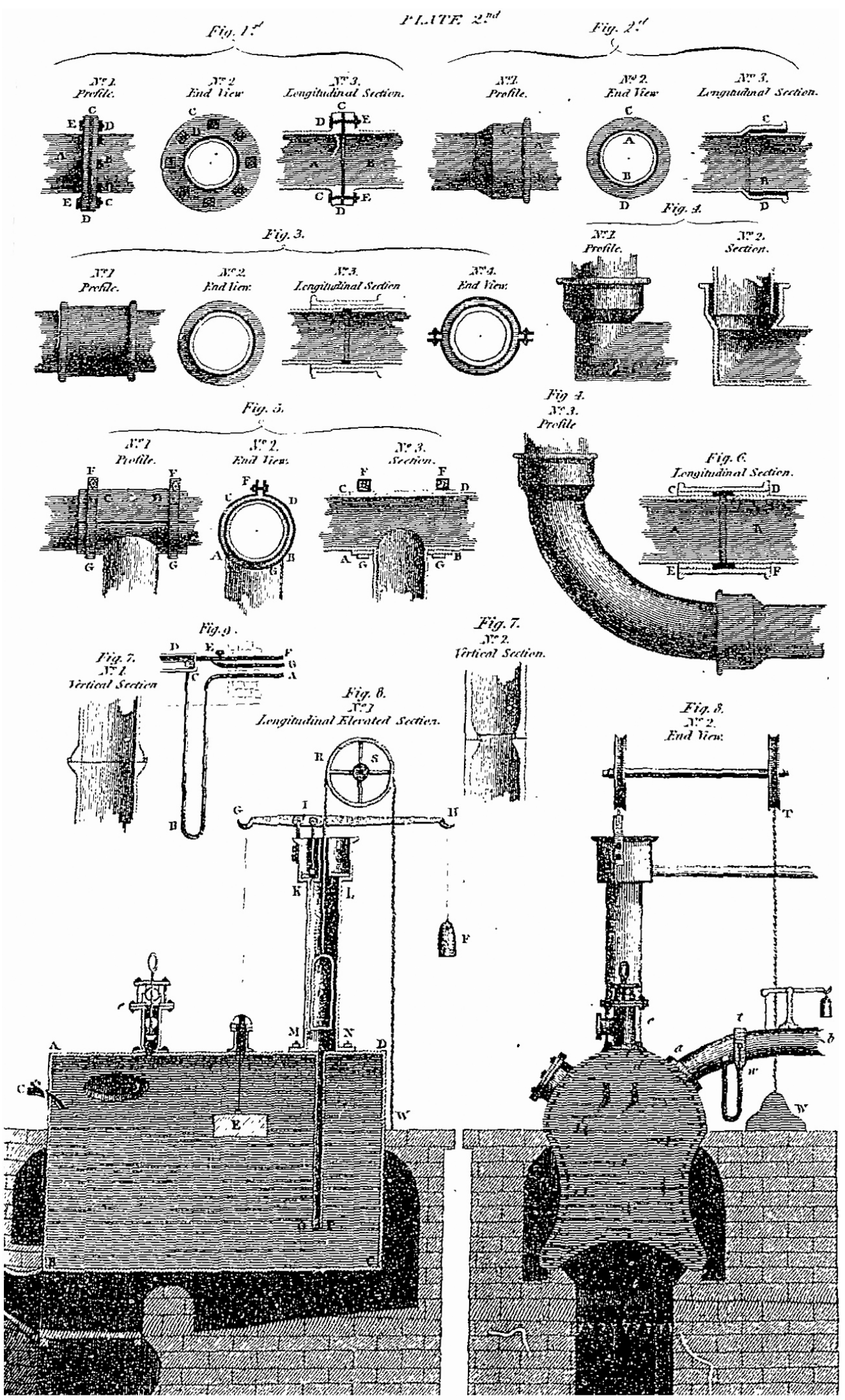

Figura 6. Robertson Buchanan, sistema de tuberías y válvulas para un sistema de calefacción central por vapor (1810). Fuente: (Buchanan 1810).

y propuso un sistema viable de válvulas (figura 6); ${ }^{4}$ una línea de desarrollo que siguió el siempre inquieto marqués de Chabannes, que en 1818 publicó una serie de dibujos al respecto, a partir de los cuales, otro ingeniero y reformador social, Thomas Tredgold, sentó los principios definitivos del diseño de calefacciones por vapor en una obra de referencia 

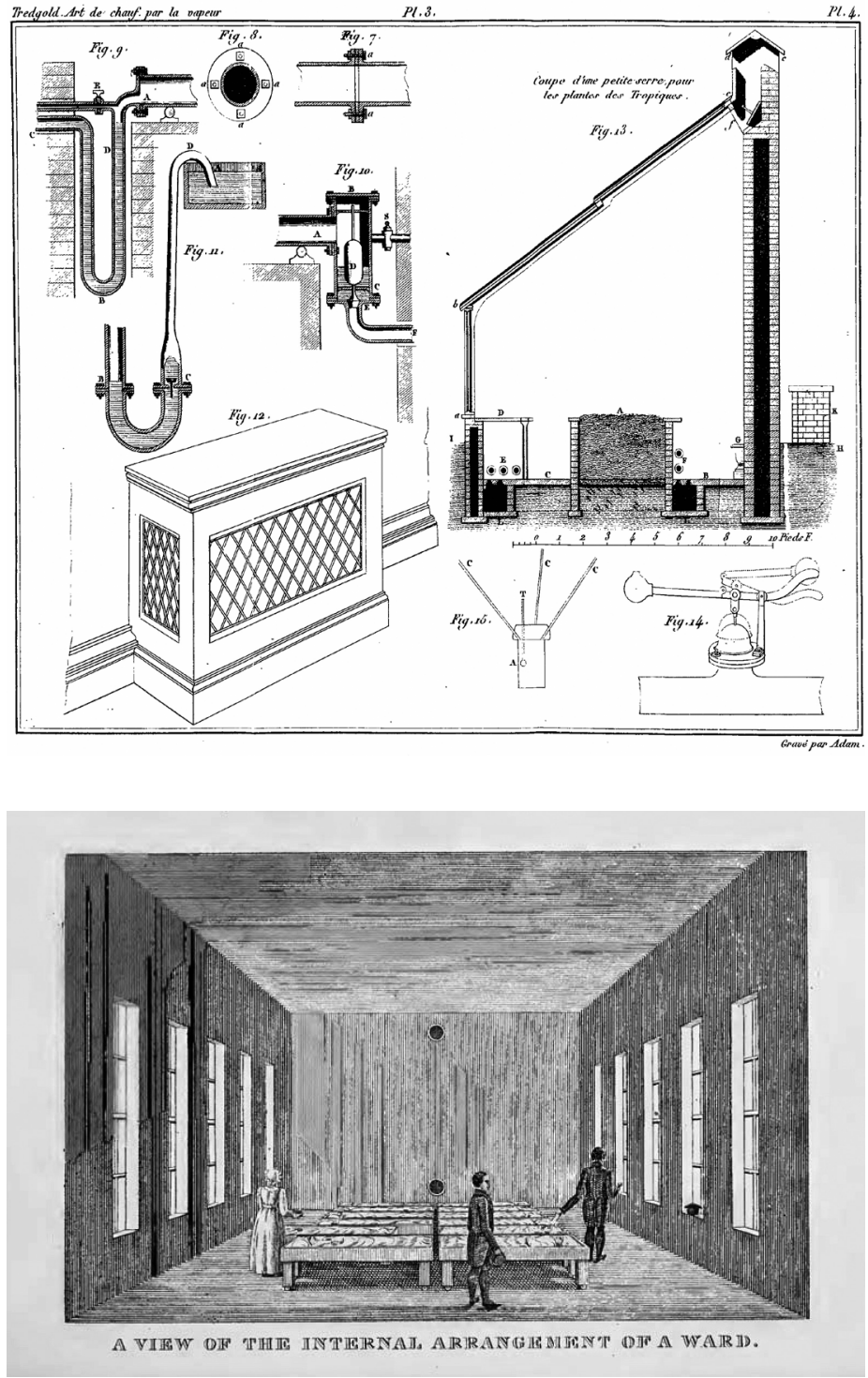

Figura 7. Thomas Tredgold, sistema de calefacción por agua caliente (1836). Fuente: (Tredgold 1836).

Figura 8. Charles Richardson, sistema de calefacción por agua caliente (1839). Fuente: (Richardson: 1839).

Figura 9. Charles Richardson, esquema de la sala tipo de un hospital ventilada y calefactada (1839). Fuente: (Richardson 1839). durante la época, Principles of Warming and Ventilating, publicada en $1824 .{ }^{5}$ Así y todo, la prometedora trayectoria de las instalaciones de vapor no dejó en ningún momento de estar amenazada por los dos grandes inconvenientes del sistema: el riesgo de condensaciones en el ambiente habitado debido a la alta temperatura del vapor de agua; y el hecho de que las altísimas presiones a las que se transmitía el vapor solian acabar reventando uniones y válvulas, cuando no simplemente provocando la explosión de la caldera.

Así las cosas, la tendencia fue usar, de un modo progresivo, instalaciones de agua caliente cuyos trazados resultaban análogos a los del vapor tanto por su planteamiento como por su eficacia, pero que no tenían sus inconvenientes, habida cuenta de que el agua fluía a una temperatura mucho más baja. Los primeros planteamientos de este sistema homotérmico fueron anecdóticos (en la déca-

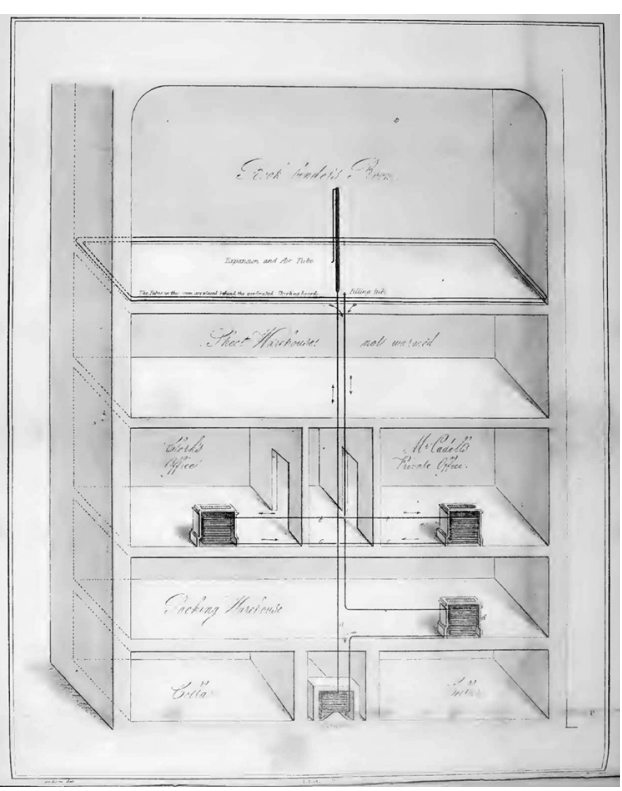

da de 1770 el jardinero Bonnemain planteó usarlo en una incubadora de pollos), y no fue hasta 1810 cuando el marqués de Chabannes propuso una versión viable para invernaderos. En los años de la preponderancia de la calefacción por vapor, los sistemas de agua caliente quedaron arrumbados, pero en 1836 Thomas Tredgold los recuperó en una nueva edición de su libro de referencia (figura 7), donde planteó una solución muy semejante a la apuntada un año después por Charles Hood, ${ }^{6}$ y que, a su vez, en 1839 sistematizó de un modo admirable Charles Richardson en un tratado con estupendos dibujos que describen toda la casuística de la colocación de los trazados y los radiadores en diferentes tipos arquitectónicos, desde invernaderos hasta inmuebles de vivienda colectiva (figuras 8 y 9). ${ }^{7}$

Eficaces a la manera un tanto precaria que fue común en la primera fase de la Revolución Industrial, todos estos sistemas no lograban resolver el principal inconveniente de la calefacción por agua: las dificultades para generar corrientes adecuadas (las bombas no se generalizarian hasta finales del siglo XIX). La solución vino de la mano del inventor Jacob Perkins, que en 1831 patentó un sistema de agua a alta temperatura que enseguida instaló en la casa de un aristócrata en Londres. Más que con el trazado o las calderas, las innovaciones de Perkins tuvieron que ver con las tuberias, que hizo estancas y continuas para eliminar en la medida de lo posible las engorrosas válvulas, y cuyo diámetro redujo para aumentar la velocidad del agua y propiciar su movimiento. Su éxito comercial 
fue tal que en los años sucesivos el sistema se colocó con éxito en multitud de edificios, algunos de ellos tan representativos como el British Museum o el palacio del duque de Wellington (Ferguson 1976: 169-171).

El triunfo de la patente de Perkins sobre el resto de los sistemas de calefacción por agua caliente supuso, a medio plazo, también el triunfo sobre las alternativas que recurrian al aire o al vapor, si bien estas siguieron gozando de cierto predicamento tanto en los edificios públicos como en los industriales, sobre todo en el entorno que, desde mediados del siglo XIX, era el más pujante del mundo en relación con los sistemas de calefacción y ventilación: el británico y el de la costa Este de los Estados Unidos. Con todo, la implantación definitiva de los sistemas centralizados, así en el desarrollado mundo anglosajón como en otros contextos, se vio limitada por un problema de índole más general, el de la mecanización efectiva de la arquitectura, que no pudo resolverse hasta que la tecnología permitió controlar, producir y distribuir otros tipos de energía más allá de los tradicionales.

Es cierto que el desarrollo de los sistemas de calefacción - con sus redes conectadas a un foco de calor separado de los espacios vivideros - hizo posible la distribución homogénea del calor -la homotermia moderna- y contribuyó a cambiar radicalmente la 'ideologia termodinámica' predominante hasta ese momento. Pero no es menos cierto que, por mucho que en el mejor de los casos estuvieran dotados de calderas, tuberias y radiadores, los edificios siguieron siendo muy precarios desde el punto de vista energético: la iluminación dependia del gas o las velas, cuando no del sebo animal; la ventilación era pobre y el aire con sus olores se remansaba en la casa, al no disponerse de medios mecánicos para moverlo; cualquier movimiento o acción, en fin, quedaba a expensas de una energía que, en lo fundamental, seguía siendo la humana. Dicho de otra manera: un ciudadano de mediados del siglo XIX podía coger un tren para viajar de Paris a Berlín y un buque a vapor para desplazarse desde Londres hasta Bombay, pero en el interior de su casa seguía teniendo que encender las velas o los quinqués uno a uno, como habian hecho sus abuelos. Así las cosas, para que pudiera llevarse a cabo con coherencia, la revolución espacial y simbólica que cabía esperar del paso de la heterotermia a la homotermia en la arquitectura necesitó que se implantara primero el que, a la postre, demostró ser el mayor invento del siglo XIX: la electricidad. En rigor, no pudo haber confort antes del enchufe.

\section{Problemas simbólicos}

La electricidad no fue la única pieza difícil de encajar en el casillero de la mecanización de la arquitectura. Desde la invención de los sistemas de calefacción centralizada y ventilación forzada hubo que hacer frente a un reto que no tenía que ver tanto con las piezas cuanto con el modo en que estas debian distribuirse en el casillero: el reto de conjugar los elementos tradicionales de la arquitectura, heredados de una concepción heterotérmica del espacio en torno al fuego abierto y la chimenea, con los nuevos sistemas que apuntaban a un nuevo espacio donde el calor se distribuía de una manera más homogénea. Se trataba de un problema a un tiempo semántico (la definición de los elementos del nuevo vocabulario), sintáctico (el establecimiento de nuevas reglas de combinación) y, en fin, también simbólico, porque acababa traduciéndose en la elección entre dos alternativas: el mantenimiento del orden compositivo heterotérmico o la creación de un nuevo orden homotérmico.

No se trataba de una elección menor. En la casa tradicional heterotérmica el hogar era el foco térmico y simbólico del espacio que organizaba las distintas partes de la habitación y las distintas partes de la casa de acuerdo al ineficaz gradiente de calor que, partiendo de la fogata encendida bajo la campana, llegaba, ya muy debilitado, hasta el limite de su alcance termico y luminoso. El resultado era una disposición basada en la cercanía al fuego, cuyos inconvenientes (los humos, las corrientes frías, el hecho de que cerca del fuego uno se sobrecalentara mientas que lejos tiritara de frío) se compensaban con la claridad tipológica y simbólica propia del modelo heterotérmico.

Con la invención de los sistemas de calefacción centralizados y con efecto térmico a distancia, esta organización se puso en entredicho: la fogata que hasta entonces había fragmentado las casas en ámbitos confortables o bien simples ámbitos de paso o almacenaje dejó paso al radiador y, con él, a la posibilidad de calentar todos los espacios domésticos de una manera homogénea. Tal posibilidad implicaba, potencialmente, vaciar de significado las relaciones de cercanía corporal y social, térmicas y a un tiempo simbólicas, fundadas en el hogar, y abrir paso a una idea de espacio homogéneo que era tan inédi- 
ta como subalterna e incómoda. Subalterna porque el espacio fluido o isótropo resultaba inviable, al menos en los climas fríos, sin una homogeneización térmica previa. E incómoda porque la ruptura de la heterotermia y, en general, de la idea del espacio focal, desactivaba las jerarquías de representación que desde siempre habian regido en la arquitectura.

El conflicto simbólico al que dio pie la homotermia fue especialmente agudo en el espacio doméstico, donde se optó por una salida de compromiso a la hora de conjugar las nuevas técnicas de calefacción con los símbolos domésticos heredados. Tal compromiso se tradujo, con mucha frecuencia, en una suerte de 'estética de la ocultación' que, como su nombre sugiere, consistió en camuflar o neutralizar visualmente el nuevo aparataje industrial, si bien esto no implicara, de ninguna manera, que los arquitectos y los ingenieros del siglo XIX no estuvieran concernidos por los problemas estéticos que implicaba el uso de las tecnologías del control del clima. Todo lo contrario: en general $-\mathrm{y}$ a diferencia de lo que parece sugerir el conocido dictum de Reyner Banham, según el cual los arquitectos del siglo XIX nunca supieron muy bien qué hacer con las máquinas-, los responsables de definir el interior burgués tuvieron éxito a la hora de cohonestar las exigencias del confort con los requisitos estéticos de su época mediante soluciones que en muchos casos fueron realmente ingeniosas. ${ }^{8}$

Las maneras en que se dio la estética de la ocultación fueron muchas. La primera, más que en una 'ocultación', consistió en un mentís absoluto a los nuevos aparatos y sistemas de calefacción y ventilación por razones de indole simbólica que, en el fondo, se sostenian en actitudes más o menos esnobs. Así, buena parte de la aristocracia inglesa se resistió, incluso entrado ya el siglo XX, a incorporar en sus mansiones radiadores, lámparas eléctricas y aun cuartos de baño, por considerarlos como cosa de 'nuevos ricos' (Rybcynski 2007: 149). Fue una actitud nostálgica y un tanto ridícula a la que, durante un tiempo, dio cierto vuelo una nueva profesión, la de 'decorador de interiores', que no dejó de ganar importancia desde mediados del siglo XIX y a la que le resultaba alérgica al uso de estufas y calefacción central, como sugiere una elocuente cita tomada de un libro de interiorismo publicado en los Estados Unidos en 1898: "El buen gusto y el savoir-vivre de los ocupantes de la casa puede deducirse de los medios que emplean para calefactarla" (Ibídem: 153). Es decir: los que consentian en instalar estufas o radiado- res en sus casas podian estar confortables en ellas, pero carecian de sensibilidad estética.

Había otras razones, más allá del esnobismo del savoir-viure, para resistirse al empleo de las nuevas tecnologías homotérmicas. Una se basaba en el prejuicio de que el aire caliente resultaba más insano que el frío, lo cual indujo a muchos reformadores sociales, incluso los más comprometidos con las nuevas tecnologias, a declararse partidarios de la chimenea tradicional, por cuanto ésta hacia compatibles el disfrute del calor con la respiración de aire fresco, tal y como creía, por ejemplo, uno de los adalides de la reforma doméstica del siglo XIX, el ya citado Thomas Tredgold (Tredgold 1824: 2-4). Otros argumentos trascendian el higienismo con razones existenciales que incidian en el papel simbólico, social y aun poético que desde siempre había desempeñado el hogar, cuyo fuego se consideraba un simbolo del carácter hospitalario de la casa; actitud que hizo suya, entre otros muchos, Henry David Thoreau en ese experimento sobre el habitar que es Walden, donde declara que "todos los hombres miran con afecto su pila de leña" (Thoreau 2005: 282), para pasar luego a lamentarse del gran vacío visual y simbólico que habian producido las nuevas tecnologías térmicas: "La estufa no solo ocupa la habitación y perfora la casa, sino que oculta el fuego: sentí que había perdido un compañero. Siempre podréis ver un rostro en las llamas" (Ibídem: 284).

No fueron éstas, sin embargo, las actitudes preponderantes a la hora de adoptar la tecnología homotérmica, sobre todo entre la burguesía adoctrinada en los ideales del progreso. En realidad, fue mucho más habitual recurrir a la segunda manera —mucho más moderada y fructifera - en que se dio la estética de la ocultación: la convivencia controlada entre los artefactos modernos y los símbolos tradicionales de la casa. En ocasiones, la convivencia se tradujo en soluciones anacrónicas, como darle a las estufas y los radiadores la forma de chimeneas góticas falsificadas o bien asimilarlos a elementos tradicionales no menos mistificados, como templetes, boiseries o escudos heráldicos (figuras 10 y 11). Estas estrategias de burdo camuflaje estilístico no tuvieron mucho recorrido, pero la actitud que las informaba no dejó nunca de estar presente en un recurso que fue habitual en la vivienda victoriana y que haría suyo, sin ningún tipo de prejuicio moderno, Frank Lloyd Wright en sus Casas de la Pradera: el recurso de duplicar las fuentes de energía de tal manera que el confort fisiológi- 


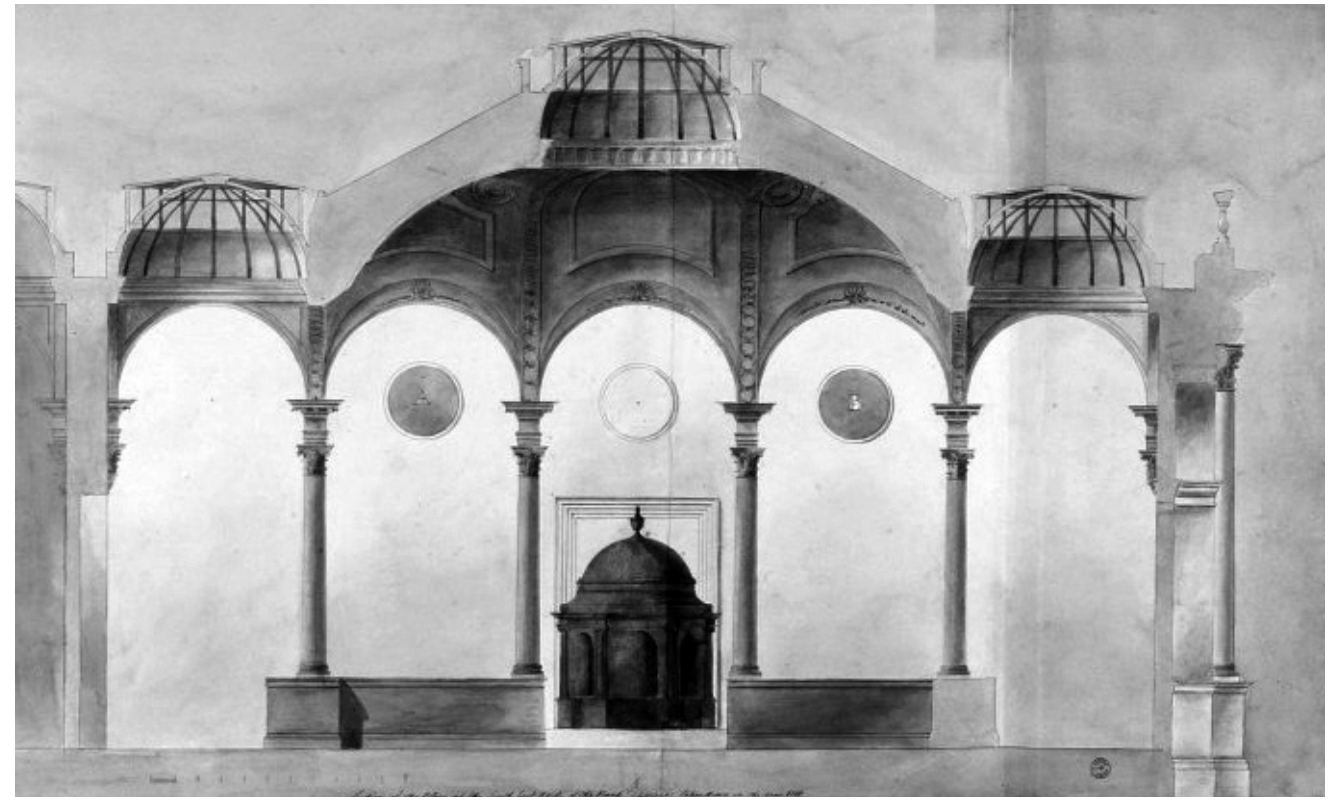

co quedara confiado a un eficiente sistema de calefacción centralizada (al fuego invisible y a distancia) en tanto el confort simbólico o ideológico seguía dependiendo de la imagen tranquilizadora y hospitalaria (asociada también al estatus social) de la gran chimenea, fuego visible y cercano en torno al cual se sentaban los miembros del hogar victoriano, igual que lo habian hecho sus antepasados en la casa tradicional.

En otras ocasiones, el anacronismo, la mistificación o la redundancia dejaban paso a un pragmatismo más cabal que, en la medida de lo posible, evitó el caer en conflictos estilísticos, formales o simbólicos, pues se sostenia en un principio de decoro tan sencillo como eficaz: en los ámbitos sin 'estatus' o 'historia', como las cocinas o los cuartos de baño, resultaba lícito mostrar con naturalidad el aparataje mecánico; pero en los demás, marcados en mayor o menor grado por su condición 'representativa', los radiadores, las tuberias y las rejillas de ventilación debian integrarse en la piel interior de la casa, ora ocultos tras pantallas decorativas, ora disimulados en falsos techos y suelos, tabiquerías dobles o plafones (figuras 12, 13 y 14). Se trataba, en rigor, de una vuelta de tuerca a la vieja idea del poché capaz de ocultar el cañón de una chimenea, las alacenas o los pasillos que permitian que los sirvientes encendieran las velas, se llevaran la ceniza del hogar o retiraran los orinales usados sin tener que encontrarse con sus señores. La estética de la ocultación dependió así de la creación de un especie de poché termodinámico y estéticamente neutro o, por decirlo con una metáfora, de una tramoya energética que hacía posible que la escena doméstica tradicional pudiera seguir representándose, pero cuyo destino, en cuanto simple espacio servidor, no era otro que permanecer oculto.

Esta estrategia era pragmática, pero también esquizofrénica, toda vez que suponía la separación tajante de la imagen del confort respecto de los artefactos que al cabo la hacian posible. Pero al mismo tiempo era tan eficaz como para introducirse rápidamente en el repertorio compositivo de la arquitectura, incardinándose en él con tal fuerza que aún hoy forma parte de la idea de una casa 'convencional'. Su rápida asimilación por el imaginario de la época se muestra, valga el ejemplo, en el modo en que, incluso en el ferrocarril o los barcos a vapor, los radiadores y las rejillas se ocultaban con cuidado en los
Figura 10. Johan Soane, estufa con forma de templete para una sala del Banco de Inglaterra (1818). Fuente: (Archivo John Soane).

Figura 11. Johan Soane, estufa con forma de escudo heráldico (1799). Fuente: (Archivo John Soane). 
Figura 12. Shirley Foster, esquema tipo de la ventilación de una casa (1883). Fuente: (Foster 1883).

Figura 13. Shirley Foster, ventilación a través de una lámpara (1883). Fuente: (Foster 1883).

Figura 14. Ventilación a través de un radiador (catálogo de la International Health Exhibition de 1884). Fuente: (International Health Exhibition 1884).
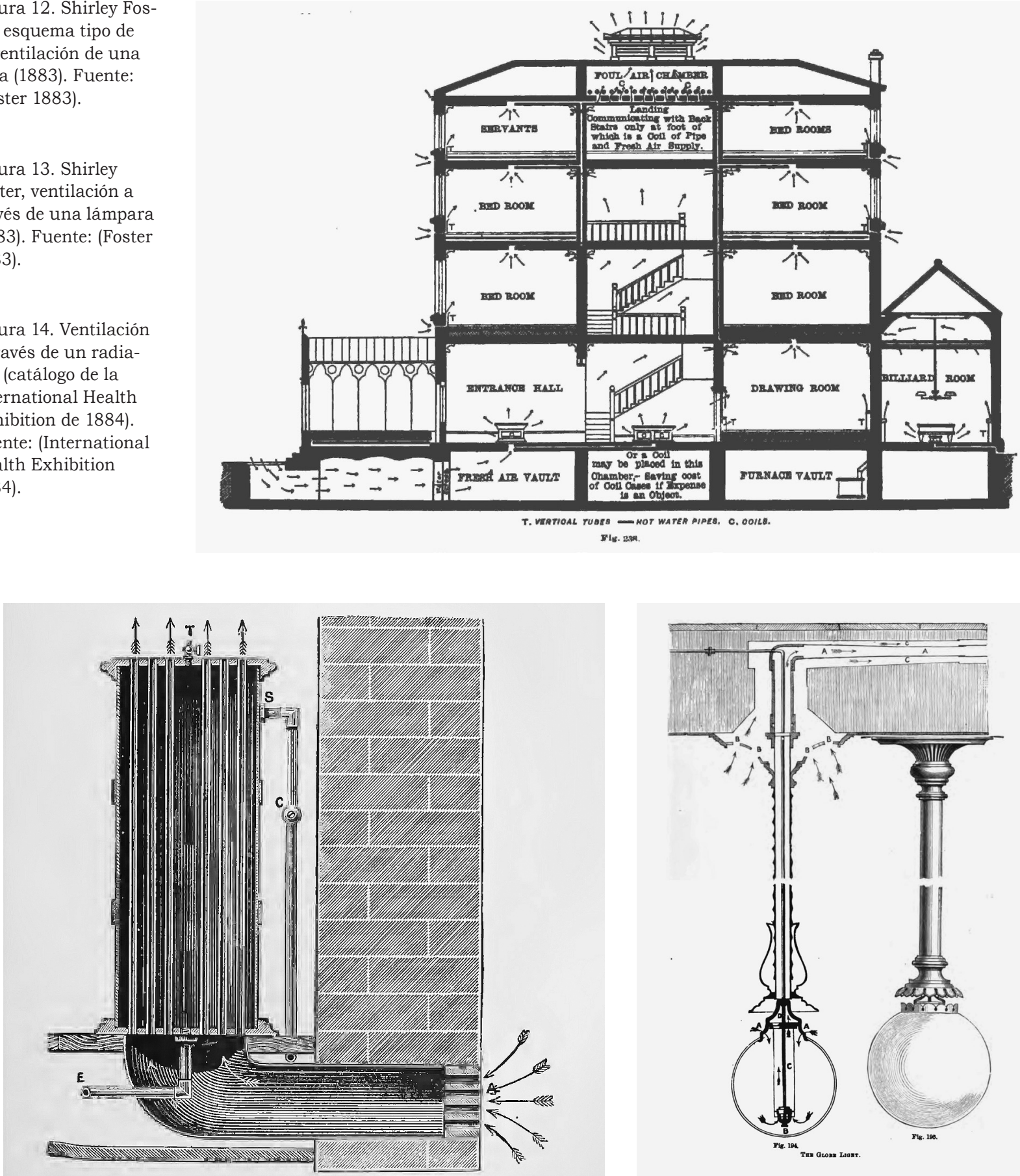

lujosos compartimentos y camarotes de primera, mientras que en los de tercera (en el caso de que estuvieran calefactados) se dejaban sin más vistos, merced a un descuido intencionado. Fue un modo de sugerir estatus que se evidencia asimismo en el célebre submarino imaginado por Julio Verne sus 20.000 leguas de viaje submarino: las ilustraciones de una de las primeras ediciones del libro, a cargo de Gustave Doré, muestran los camarotes más formales del submarino -el salón y la biblioteca del Capitán Nemocomo espacios indistinguibles de los de cual-

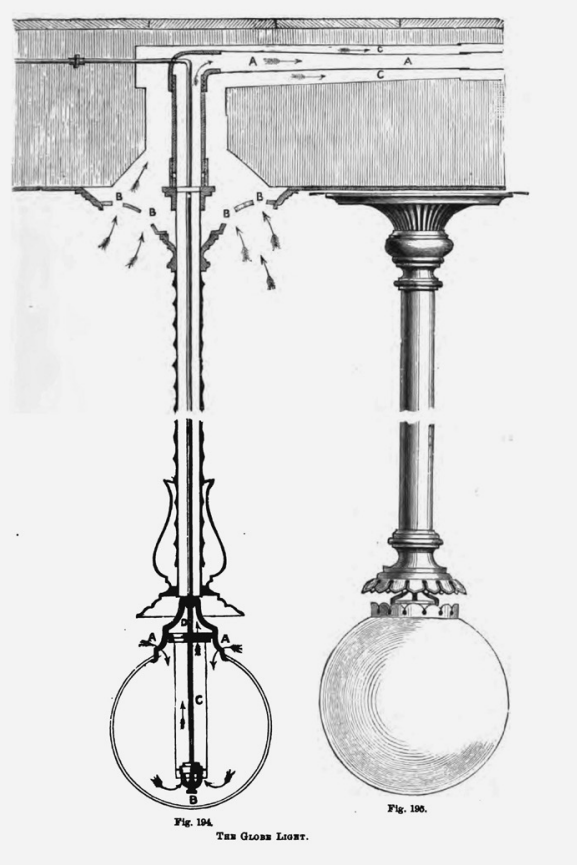

quier casa burguesa acomodada, por mucho que tal salón y biblioteca estén servidos por una maquinaria sofisticada y perfecta que queda oculta en los cuartos técnicos, y por mucho también que estén contenidos en el vientre de un inmenso artefacto que atraviesa con arrogancia los mares. El Capitán Nemo, como cualquier burgués de su época, se avergonzaba de sus máquinas cuando tenía que atender a sus invitados en el salón de recibir del Nautilus (figura 15).

La llegada de la modernidad maquinista cambiaría un tanto las tornas en la medida 
en que matizó la estética de la ocultación que habian construido con tanto cuidado los arquitectos e ingenieros del siglo XIX. Las vanguardias, sobre todo las futuristas, comenzaron a considerar la máquina menos por sus efectos utilitarios que por su capacidad para dar cumplida cuenta de una época definida por la tecnología —el Zeitgeist mecánico-, y este cambio de perspectiva hizo que los radiadores, los conductos, las rejillas y, con ellos, también los nuevos materiales como el acero y el hormigón armado, fuesen llamados a cumplir designios estéticos (Prieto 2016: 113). Sin embargo, y por mucha que fuera la fuerza que tuvo durante un tiempo la retórica del maquinismo, los artefactos de calefacción y ventilación no desempeñaron a la postre papeles simbólicos mucho más ambiciosos que los que se les habian adjudicado durante la época victoriana. En rigor, las estrategias modernas apenas trascendieron la mera mostración selectiva de algunos artefactos: si en la casa victoriana el radiador se había ocultado tras una pantalla de madera o chapa, en la casa moderna se intentaba presentar con naturalidad; si antes el lavabo de cerámica blanca había quedado confinado en el retrete, ahora se procuraba exhibir sin complejos en las habitaciones de 'aparato' o incluso en los vestíbulos 'de recibir'.

Pero tales atrevimientos (característicos asimismo de esa segunda oleada tecnocrática que seria el high tech de la década de 1970) no dejaban de ser migajas simbólicas que apenas conseguian dar cuenta del papel fundamental pero latente que los sistemas de calefacción habían desempeñado a la hora de configurar los ambientes homogéneos en lo térmico e isótropos en lo espacial de la modernidad. En los años de las vanguardias, y más allá de la fascinación formal por la máquinas, pocos fueron los arquitectos conscientes de este papel, y así, una vez pasados los furores vanguardistas, tanto los radiadores como las tuberías (y, desde mediados del siglo XX, asimismo los aparatos y las rejillas de aire acondicionado) volvieron a ser tratados con los mismos mecanismos estéticos que habian impuesto, por mor de la idea de decoro, los ingenieros y arquitectos victorianos, aunque con mucha menor sutileza. Con el retour à l'ordre experimentado por la arquitectura tras la Segunda Guerra Mundial, la vieja estética de la ocultación volvió, de hecho, por sus fueros, con una ambición que resultó incluso mayor que la que había demostrado tener antes, y todo ello de la mano del que cabe considerar uno de los mayores inventos
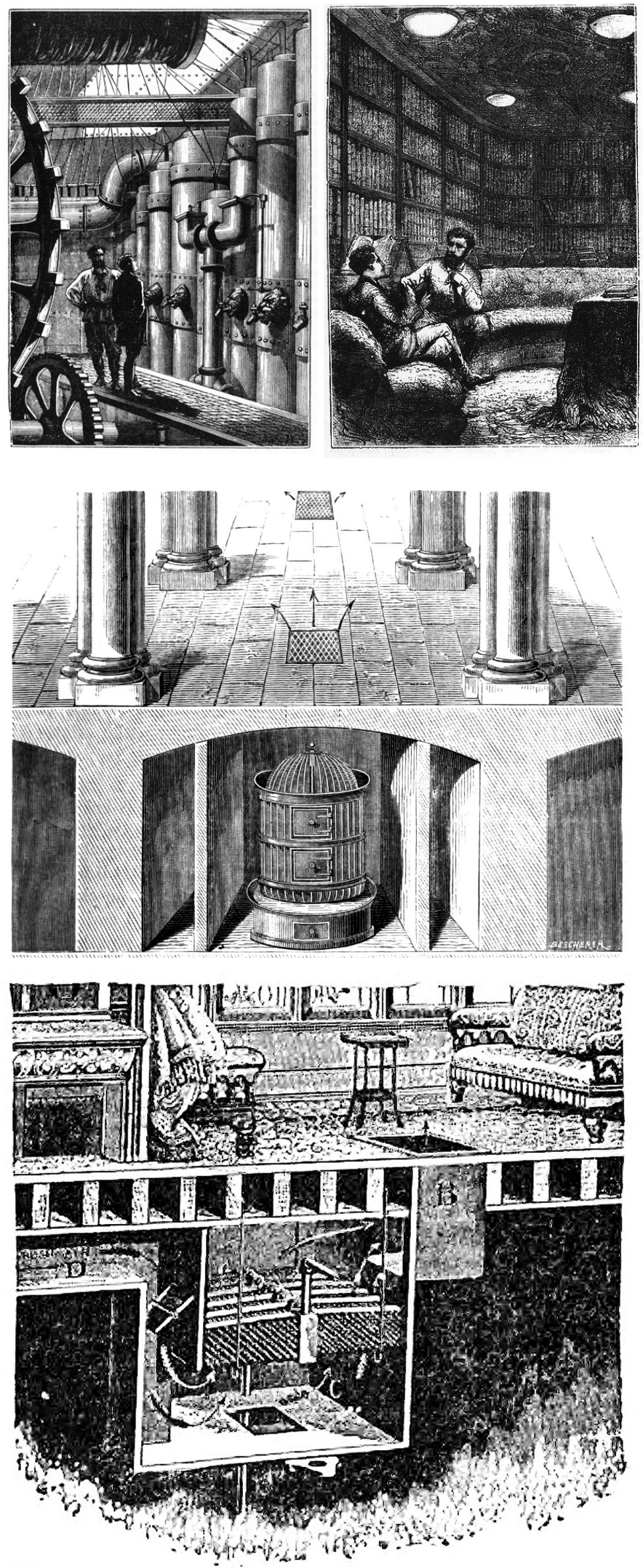

de la arquitectura moderna: el techo técnico, evolución de los falsos techos y plafones victorianos.

En rigor, el falso techo devenido techo técnico, cámara a medio camino entre lo visible 

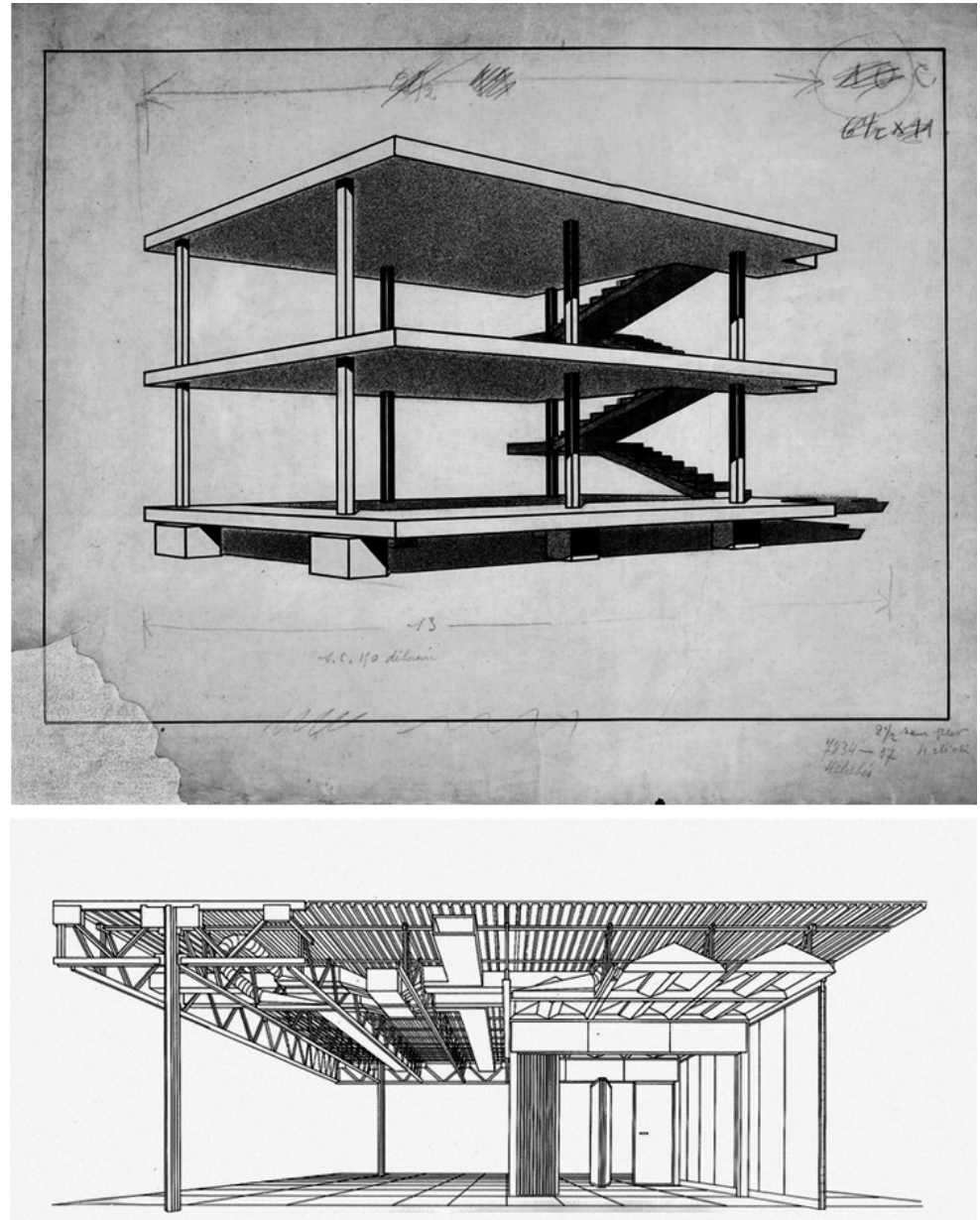

Figura 15. La sala de máquinas y el salón del capitán Nemo en el Nautilus, según Doré (1873). Fuente: (Verne/ Doré 1873).

Figura 16. Estufa integrada en el suelo, según Joly (1873), y sistema enterrado de ventilación mecánica, según Carpenter (1900). Fuente: (Joly 1873) y (Carpenter 1900).

Figura 17. El sistema Dom-ino de Le Corbusier (1914) y el Sistema SCSD de Ezra Eherenkranz (1960). Fuente: (Fondation Le Corbusier) e (Internet). y lo invisible, entre el espacio representativo y el de servicios, no fue sino una versión más pragmática y también más burda del poché termodinámico victoriano (figura 16). Más pragmática porque permitió resolver con mayor libertad las redes de instalaciones cada vez más extensas y complejas que las comenzaron a exigir desde la segunda mitad del siglo XX. Pero también más burda, en la medida en que el techo técnico, en sus versiones más adocenadas (que fueron también las más frecuentes) arrumbó la complejidad y la sutileza estética a la hora de integrar radiadores, rejillas y conductos que había sido propia de la mejor arquitectura higienista decimonónica. Asi y todo, el falso techo integrado en la red general de instalaciones modernas no dejó de ser un elemento útil y poderoso, que reflejó el cambio de paradigma desde la modernidad canónica, centrada en las poéticas de los nuevos materiales y en la composición purista, hasta la modernidad madura, obsesionada por la flexibilidad funcional y el confort. Un cambio del que se puede dar cuenta comparando dos ejemplos característicos de normativas técnicas y la cultura del confort uno y otro paradigma: de un lado, el Sistema Dom-ino de Le Corbusier, pergeñado en 1914, verdadero principio tectónico de la arquitectura moderna; del otro, el Sistema SCSD diseñado por Ezra Eherenkranz a principios de la década de 1960, materialización del giro termodinámico que ha experimentado la arquitectura desde aquellos años hasta ahora (figura 17).

\section{Notas}

1 Sylvester, Charles, The Philosophy of Domestic Economy; as exemplified in the mode of warming, ventilating, washing, drying, \& cooking, and in Various Arrangements contributing to the Comfort and Convenience of Domestic Life, adopted in The Derbyshire General Infirmay, and more recently, on a greatly extended scale, in several other public buildings, newly erected in this country, Londres: H. Barnett, High Strett, 1819. Se trata quizá del primer tratado dedicado al confort en la arquitectura moderna, que sentó las bases de los muchos que se es escribieron a lo largo del siglo XIX.

2 El artículo de Bruegmann es un excelente resumen de la historia de los sistemas de calefacción centralizada. Sobre el particular, véase también Peterson, Charles E., Building Early America: Contributions toward the History of a Great Industry, Rednor: Chilton Book Company, 1976, pp. 165-185.

3 Transactions of the Society of Arts, 1806, pp. 150-162.

4 Buchanan, Robertson, An Essay of the Warming of Mills and Other Buildings by Steam, Glasgow, 1807.

5 Tredgold, Thomas, Principles of Warming and Ventilating Public Buildings, Dwelling Houses, Manufactories, Hospitals, Hot Houses, Conservatories, etc, Londres, 1824.

6 Hood, Charles, A Practical Treatise of Warming Buildings by Hot Water, Londres: Whittaker \& Co., 1837.

7 Richardson, Charles J., A Popular Treatise on the Warming and Ventilation of Buildings; showed the advantages of the improved system of heated water circulation, Londres: John Weale, architectural library, 1839.

8 Tanto estas soluciones ingeniosas como la idea que los arquitectos del siglo XIX tenian de 'su época' le parecian a Reyner Banham - uno de los pioneros en abordar estos temas-ideológica y estéticamente insuficientes por no ser lo bastante 'modernas' o 'maquinistas' (desde la perspectiva hegeliana de Banham, que es la perspectiva del 'a toro pasado', resulta fácil emplear a discreción las etiquetas 'antiguo', 'moderno' y 'apropiado' para una 'época'). Esta actitud de partida se traduce en el modo tan insuficiente con que, en The Architecture of Well-tempered Environment, Banham trata los problemas de la introducción de la calefacción centralizada y los sistemas de ventilación en la arquitectura del siglo XIX; una insuficiencia que puede explicarse con dos razones: que el británico desconociera 
el contenido de los muchísimos volúmenes que se publicaron en la época victoriana en relación con las nuevas tecnologías y su integración en la arquitectura; o que, simplemente, prefiera omitirlos para sostener, desde una perspectiva histórica adulterada, sus propios argumentos estetico-tecnocráticos. Presentados en textos de variada condición, estos argumentos se reducen, en puridad, a uno solo al que cabe darle la forma de un silogismo: 'La arquitectura debe expresar el Espíritu de la Época; el Espiritu de la Época es el maquinismo; ergo los edificios deben parecerse a las máquinas'. En este sentido, el gran especialista en historia de la calefacción Robert Bruegmann critica a Banham con las siguientes palabras: "[His] point of view obscures many of the major goals of 19th-century architects (...) It becomes apparent that 19th-century architects and engineers frequently succeded in integrating aesthetic considerations with a concern for environmental control" (Bruegmann 1978: 154).

\section{Bibliografia}

Banham, Reyner. 1969. The Architecture of Well-tempered Environment, Londres: Architectural Press, 1969 [edición en español: La arquitectura del entorno bien climatizado, Buenos Aires: Ediciones Infinito, 1975].

Bernan, Walter. 1845. On the History and Art of Warming and Ventilating Rooms and Buildings, vol. 1, Londres: George Bell.

Bernan, Walter. 1845. On the History and Art of Warming and Ventilating Rooms and Buildings, vol. 2, Londres: George Bell.

Bruegmann, Robert. 1978. "Central Heating and Forced Ventilation: Origins and Effects on Architectural Design", Journal of the Society of Architectural Historians, vol. $37, \mathrm{n}^{\circ} 3$ (octubre 1978).

Buchanan, Robertson. 1807. An Essay of the Warming of Mills and Other Buildings by Steam, Glasgow.

CARpenter, Rolla. 1900. Heating and Ventilating Buildings: A Manual for Heating Engineers and Architects, Nueva York: John Wiley and Sons.

Chabannes, Jean-Baptiste de. 1818. On conducting air by forced ventilation and regulating the temperatures in dwellings, with a description of the application of the principles as established in Covent Garden Theatre and Lloyd's Subscription Rooms, Londres.

Chabannes, Jean-Baptiste de. 1820. Appendix to the Marquis de Chabannes' publication of Conducting air by forces ventilation and equalizing the temperature of dwellings, Londres.

Evans, Oliver. 1795. The Young Mill-Wright \& Miller's Guide, Filadelfia.

Forster, Shirley. 1883. Our Homes and How to Make Healthy, Londres: Cassell \& Company.

Giedion, Sigfried. 1948. Mechanization Takes Command: A Contribution to Anonymous History, Nueva York: Oxford University Press.
Hood, Charles. 1837. A Practical Treatise of Warming Buildings by Hot Water, Londres: Whittaker \& Co.

International Health Exhibition London 1884. The Health Exhibition Literature. Vol. 1: Health in the Dwelling, Londres: William Clowes and sons.

Joly, V. Ch. 1873. Traité pratique du chauffage, de la ventilation et de la distribution des eaux dans les habitations particulières, París: J. Baudry.

Peterson, Charles E. 1976. Building Early America: Contributions toward the History of a Great Industry, Rednor: Chilton Book Company.

PRIETO, Eduardo. 2016. La ley del reloj: arquitectura, máquinas y cultura moderna, Madrid: Ediciones Cátedra.

Richardson, Charles J. 1839. A Popular Treatise on the Warming and Ventilation of Buildings; showed the advantages of the improved system of heated water circulation, Londres: John Weale, architectural library.

RyBCynski, Witold, 2007. La casa: historia de una idea, San Sebastián: Nerea [primera edición en inglés: The Home: A Short History of an Idea, Londres: Penguin Books, 1987].

Sylvester, Charles. 1819. The Philosophy of Domestic Economy; as exemplified in the mode of warming, ventilating, washing, drying, \& cooking, and in Various Arrangements contributing to the Comfort and Convenience of Domestic Life, adopted in The Derbyshire General Infirmay, and more recently, on a greatly extended scale, in several other public buildings, newly erected in this country, Londres: H. Barnett, High Strett.

Tann, Jennifer. 1970. The Development of the Industry, Londres: Conmarket Press.

Thoreau, Henry D. 2005. Walden, Madrid: Cátedra. Transactions of the Society of Arts, 1806 (150-162).

TREDGold, Thomas. 1824. Principles of Warming and Ventilating Public Buildings, Dwelling Houses, Manufactories, Hospitals, Hot Houses, Conservatories, etc. Londres.

WRIGHT, Lawrence. Home Fires Burning: The History of Domestic Heating and Cooking, Londres: Routledge \& Kegan Paul, 1964.
Fecha final recepción artículos: 25/04/2019

Fecha aceptación: 26/06/2019

Artículo sometido a revisión por dos revisores independientes por el método doble ciego. 